\title{
Reliability of the application of transvaginal color Doppler ultrasound in the identification of pelvic tumors in women of childbearing age
}

\author{
Mingchun Zhi ${ }^{1}$, Miaoqian Wang ${ }^{1}$, Wei $\mathrm{Li}^{1}$, Lijie $\mathrm{Ma}^{1}$, Qian $\mathrm{Liu}^{2}$, Ye $\mathrm{Li}^{1}$, Qiubo $\mathrm{Lv}^{1}$ \\ ${ }^{1}$ Department of Obstetrics and Gynecology, Beijing Hospital, National Center of Gerontology; Institute of Geriatric Medicine, Chinese Academy \\ of Medical Sciences, Beijing, China; ${ }^{2}$ National Center for Clinical Laboratories, Beijing Hospital, National Center of Gerontology; Institute of \\ Geriatric Medicine, Chinese Academy of Medical Sciences, Beijing, China \\ Contributions: (I) Conception and design: M Zhi, Q Lv; (II) Administrative support: M Wang; (III) Provision of study materials or patients: M Zhi, M \\ Wang, W Li, L Ma, Q Liu; (IV) Collection and assembly of data: All authors; (V) Data analysis and interpretation: W Li, L Ma, Q Liu, Y Li, Q Lv; (VI) \\ Manuscript writing: All authors; (VII) Final approval of manuscript: All authors. \\ Correspondence to: Qiubo Lv. Gynecology and Obstetrics Ultrasound Room, Second Floor of the Beijing Hospital Outpatient Clinic, No. 1, Dongdan \\ Dahua Road, Dongcheng District, Beijing 100730, China. Email: qiubo2185@163.com.
}

Background: Recently, transvaginal color Doppler ultrasound (TVCDU) has been widely used in the diagnosis of pelvic tumors. This study aimed to assess the reliability of the application of TVCDU in the identification of pelvic tumors in women of childbearing age.

Methods: The clinical data of 209 patients with pelvic tumors at childbearing age admitted to our hospital from January 2016 to December 2019 were retrospectively analyzed. The ultrasound signs, color Doppler ultrasound score (CDUS), and resistance index (RI) of benign and malignant pelvic tumors diagnosed by TVCDU were analyzed. The value of transabdominal color Doppler ultrasound (TACDU) and TVCDU in the diagnosis of benign and malignant pelvic tumors was calculated.

Results: There were 150 cases with benign pelvic tumors and 59 cases with malignant tumors. Most benign tumors had an intact capsule and regular shape, were mainly cystic, and were rarely accompanied by ascites; meanwhile, malignant tumors were mostly non-capsular and irregular in shape, mainly solid or cystic, and were often accompanied by ascites. There were significant differences in the above-mentioned ultrasound signs between benign and malignant tumors $(\mathrm{P}<0.05)$. The CDUS score of benign pelvic tumors was significantly lower than that of malignant tumors, and the RI value was significantly higher than that of the malignant tumors $(\mathrm{P}<0.05)$. The sensitivity, specificity, and accuracy of TACDU in the diagnosis of benign pelvic and malignant tumors were $83.33 \%, 84.75 \%$, and $83.73 \%$, respectively; and the sensitivity, specificity, and the accuracy of TVCDU in the diagnosis of benign pelvic and malignant tumors were $95.33 \%, 88.14 \%$, and $93.30 \%$, respectively; thus TVCDU had a superior performance compared to TACDU, especially in sensitivity and accuracy $(\mathrm{P}<0.05)$.

Conclusions: The sensitivity and accuracy of TVCDU in the differential diagnosis of benign pelvic and malignant tumors in women at childbearing age were significantly higher than those of TACDU. The combined application of CDUS and RI can further improve the accuracy in the diagnosis of pelvic tumors.

Keywords: Transvaginal color Doppler ultrasound (TVCDU); pelvic tumors; benign and malignant; women of childbearing age

Submitted Oct 26, 2020. Accepted for publication Dec 04, 2020.

doi: $10.21037 / \mathrm{atm}-20-7406$

View this article at: http://dx.doi.org/10.21037/atm-20-7406 


\section{Introduction}

The majority of pelvic tumors originate from the female reproductive system, which are common lesions in women of childbearing age, and generally have no obvious clinical symptoms (1). If the diameter of the pelvic tumor is less than $10 \mathrm{~cm}$ and does not exceed the range of the pelvis, the patient can hardly feel it, nor can a doctor easily detect it when performing an abdominal palpation. Indeed, this type of tumor can only be diagnosed by pelvic examination $(2,3)$. B-ultrasound is the first choice for gynecological diseases because it is easy to operate, quick, and can accurately display the location, size, texture, and the relationship of any pelvic tumors with the surrounding tissues (4). Transabdominal color Doppler ultrasound (TACDU) has traditionally been one of the most commonly used clinical diagnostic methods. However, abdominal ultrasound scanning is often hampered by interference from intestinal gas and bladder filling, while the resolution of the distant field of ultrasonic probe lacks quality, which limits the utility of the imaging of the internal fine anatomical structure (5). With the continuous advancement and development of imaging technology, transvaginal color Doppler ultrasound (TVCDU) has seen an increased and more widespread use in the diagnosis of pelvic tumors. Compared with transabdominal ultrasound, the probe resolution of TVCDU is higher, and the diagnosis of the lesions and blood flow of the internal organs of the pelvis is easier, as well as no need to hold the urine (6). In this study, the reliability of TVCDU for identifying benign and malignant pelvic tumors in women of childbearing age was evaluated. We present the following article in accordance with the STARD reporting checklist (available at http:// dx.doi.org/10.21037/atm-20-7406).

\section{Methods}

\section{General information}

The clinical data of pelvic tumor patients at childbearing age admitted to our hospital from January 2016 to December 2019 were retrospectively analyzed. The inclusion criteria were the following: (I) patients who had passed the ultrasound examination, and were then confirmed to be with pelvic tumor by surgery, laparoscopy, gastrointestinal endoscopy, or histopathological examination; (II) patients with complete clinical data, without loss or defect; (III) female (IV) patients with malignant tumors who did not receive chemotherapy or radiotherapy before surgery.
(V) patients at childbearing age of 15-50 years old. The exclusion criteria were the following: (I) patients with other malignant diseases; (II) patients in a pregnancy or lactation period; (III) patients with ultrasound examination contraindications; (IV) patients with congenital diseases or immune dysfunction; (V) patients were contraindicated for a second surgery. This study was approved by the Beijing Hospital (No. 2019BJYYEC-167-01). All procedures performed in this study involving human participants were in accordance with the Declaration of Helsinki (as revised in 2013). Individual consent for this retrospective analysis was waived.

\section{Detection method}

The E10 color Doppler ultrasound scanner (GE Healthcare Systems, USA) was used for examination, with the abdominal ultrasound examination using a 3.5 $\mathrm{mHZ}$ abdominal probe. Before the examination, patients were instructed to drink a copious amount of water to fill their bladder. Two-dimensional ultrasound was used to observe and record the size, location, nature, internal echo, and the presence or absence of capsules of the mass through horizontal, vertical, and oblique multidirectional observation. After the abdominal ultrasound was completed, patients were asked to empty their bladder and to assume the lithotomy position for transvaginal ultrasound diagnosis with a $7.5 \mathrm{MHz}$ vaginal probe. A disposable condom was used to cover the vaginal probe, and the inside and outside of the cover was coated with a disinfectant coupling agent. Multisection scans were then performed to carefully observe the patient's pelvic cavity, and color Doppler ultrasound was used to detect the blood flow of the lesion.

\section{Observation indicators}

The ultrasound signs were collected using TVCDU and CDUS, and the resistance index (RI) of benign and malignant pelvic tumors were analyzed. With the "gold standard" of pathological diagnosis, the value of TACDU and TVCDU in the diagnosis of pelvic benign and malignant tumors was compared. The color Doppler scoring criteria for pelvic tumors are shown in Table 1 .

\section{Statistical methods}

The data in this study were statistically analyzed by SPSS 22.0 software (IBM, NY, USA). The measurement data are 
Table 1 Color Doppler score criteria for pelvic tumors

\begin{tabular}{lcccccc}
\hline Score & $\begin{array}{c}\text { Tumor } \\
\text { surface }\end{array}$ & $\begin{array}{c}\text { Tumor thickness } \\
(\mathrm{mm})\end{array}$ & $\begin{array}{c}\text { Tumor } \\
\text { separation }\end{array}$ & Tumor echo & $\begin{array}{c}\text { Blood flow } \\
\text { characteristics }\end{array}$ & Blood flow distribution \\
\hline 1 & Smooth & $\leq 3$ & None & No reflected echo & None & Pene \\
2 & Irregular & $4-5$ & A few $(\leq 3)$ & $\begin{array}{c}\text { No reflective or liquid } \\
\text { turbidity area }\end{array}$ & $\begin{array}{c}\text { Stellate } \\
\text { punctiform }\end{array}$ \\
3 & Papillary & $>6$ & Many $(>3)$ & A little strong echo & Short-linear & Peripheral + separation \\
4 & Disorder & & & Strong echo & Strip & Center \\
5 & & & Mixed echo accompanied & Reticulate & Peripheral + center \\
\hline
\end{tabular}

A score $\geq 4.0$ was diagnosed as a pelvic tumor, which included malignant tumors.

Table 2 Pathological types of tumors

\begin{tabular}{lcc}
\hline Pathological types & Case (n) & Percentage (\%) \\
\hline Benign ( $\mathrm{n}=150)$ & 12 & 8.00 \\
Benign ovarian Brenner tumor & 21 & 14.00 \\
Chocolate cyst & 49 & 32.67 \\
Ovarian serous cyst & 38 & 25.33 \\
Endometrial implantation cyst & 30 & 20.00 \\
Mucinous cystadenoma & & \\
Malignant (n=59) & 23 & 38.98 \\
Ovarian serous adenocarcinoma & 16 & 27.12 \\
Mucinous ovarian adenocarcinoma & 20 & 33.90 \\
Cervical cancer & & \\
\hline
\end{tabular}

described by the mean \pm standard deviation $(\bar{x} \pm s)$, and were analyzed by $t$ test. The count data are expressed as pass rates or composition ratios, and were analyzed by chi-squared $\left(\chi^{2}\right)$ test. Results with $\mathrm{P}$ value $<0.05$ were considered to be statistically significant.

\section{Results}

The specific pathological types of tumors of the involved patients

After selection based on the inclusion and exclusion criteria, a total of 209 patients were enrolled, all of whom were female, aged 24 to 52 years old, with an average age of $30.66 \pm 7.86$ years old. The main clinical symptoms of the enrolled patients included menorrhagia, prepubertal or postmenopausal lump with uterine bleeding, acute abdominal pain in the lower abdomen, periodic abdominal pain, fever, and abdominal distension. Among these patients, there were 150 cases of benign pelvic tumors and 59 cases of malignant tumors. The specific pathological types of tumors are shown in Table 2.

\section{Ultrasound signs of pelvic benign and malignant tumors}

Most benign tumors had a complete capsule and regular morphology, were mainly cystic, and were rarely accompanied by ascites; meanwhile, malignant tumors were mostly non-encapsulated with irregular morphology, mainly solid or cystic, and were often accompanied by ascites. The differences of the above-mentioned ultrasound signs between benign and malignant tumors were significant $(\mathrm{P}<0.05$, Table 3).

\section{Comparison of CDUS score and RI value of benign and pelvic malignant tumors}

The CDUS score of benign pelvic tumors was significantly lower than that of malignant tumors, and the RI value of benign pelvic tumors was significantly higher than that of malignant tumors $(\mathrm{P}<0.05$, Table 4$)$.

\section{Comparison of TACDU and TVCDU in the pathological diagnosis of benign and malignant pelvic tumors}

Among the 209 patients, a total of 175 cases were detected by TACDU, with a total diagnosis rate of $83.73 \%$, and a total of 195 cases were detected by TVCDU, with a total diagnosis rate of $93.30 \%$, which was significantly higher than that of TACDU $\left(\chi^{2}=9.414, \mathrm{P}=0.002\right.$, Table 5). 
Table 3 Ultrasound signs of benign and malignant pelvic tumors [n (\%)]

\begin{tabular}{lllllll}
\hline Group & Encapsulated & Regular morphology & Cystic & Solid & Cyst-solid & Ascites \\
\hline Benign $(\mathrm{n}=150)$ & $140(93.33)$ & $124(82.67)$ & $106(70.67)$ & $17(11.33)$ & $26(17.33)$ & $4(2.67)$ \\
Malignant $(\mathrm{n}=59)$ & $21(35.59)$ & $13(22.03)$ & $16(27.12)$ & $23(38.98)$ & $22(37.29)$ & $32(54.24)$ \\
$\chi^{2}$ & 79.795 & 68.937 & 33.048 & 20.918 & 9.531 & 78.985 \\
$P$ & 0.001 & 0.001 & 0.001 & 0.001 & 0.002 & 0.001 \\
\hline
\end{tabular}

Table 4 Comparison of CDUS scores and RI values between benign and malignant pelvic tumors $(\bar{x} \pm s)$

\begin{tabular}{lcc}
\hline Group & CDUS score & Rl \\
\hline Benign $(n=150)$ & $2.78 \pm 1.11$ & $0.52 \pm 0.07$ \\
Malignant $(n=59)$ & $6.14 \pm 2.23$ & $0.36 \pm 0.03$ \\
$t$ & 14.479 & 16.936 \\
$P$ & 0.001 & 0.001 \\
\hline
\end{tabular}

CDUS, color Doppler ultrasound score; RI, resistance index.

Table 5 Comparison of TACDU and TVCDU in pathological diagnosis [n (\%)]

\begin{tabular}{lccc}
\hline \multirow{2}{*}{ Detection method } & \multicolumn{2}{c}{ Pathologic diagnosis } & \multirow{2}{*}{ Total } \\
\cline { 2 - 3 } & Benign & Malignant & \\
\hline TACDU & $125(83.33)$ & $9(15.25)$ & $134(64.11)$ \\
Benign & $25(16.67)$ & $50(84.75)$ & $75(35.89)$ \\
Malignant & & & \\
TVCDU & $143(95.33)$ & $7(11.86)$ & $150(71.77)$ \\
Benign & $7(4.67)$ & $52(88.14)$ & $59(28.23)$ \\
\hline
\end{tabular}

TACDU, transabdominal color Doppler ultrasound; TVCDU, transvaginal color Doppler ultrasound.

Table 6 Comparison of sensitivity and specificity between TACDU and TVCDU in the diagnosis of benign and malignant pelvic tumors (\%)

\begin{tabular}{lccc}
\hline $\begin{array}{l}\text { Detection } \\
\text { method }\end{array}$ & Sensitivity & Specificity & Accuracy \\
\hline TACDU & $83.33(125 / 150)$ & $84.75(50 / 59)$ & $83.73(175 / 209)$ \\
TVCDU & $95.33(143 / 150)$ & $88.14(52 / 59)$ & $93.30(195 / 209)$ \\
$\chi^{2}$ & 11.334 & 0.289 & 9.414 \\
$P$ & 0.001 & 0.591 & 0.002 \\
\hline
\end{tabular}

TACDU, transabdominal color Doppler ultrasound; TVCDU, transvaginal color Doppler ultrasound.
Comparison of sensitivity and specificity of TACDU and TVCDU in the diagnosis of pelvic benign and malignant tumors

The sensitivity, specificity, and accuracy of TACDU in diagnosing benign and malignant pelvic tumors were $83.33 \%, 84.75 \%$, and $83.73 \%$, respectively. The sensitivity, specificity, and accuracy of TVCDU in diagnosing benign and malignant pelvic tumors were $95.33 \%, 88.14 \%$, and $93.30 \%$ respectively, which were all significantly higher than those of TACDU $(\mathrm{P}<0.05$, Table 6).

\section{Analysis of two cases}

Physical examination of a 45-year-old female patient revealed a left accessory mass that had persisted for over 1 month, who was shown to be with ovarian serous adenocarcinoma, moderately differentiated (Figure 1). And another patient was a 44-year-old woman who complained of dull pain in her lower abdomen for 20 years, accompanied by menstrual changes and frequent urination for 2 months, who was shown to be with pelvic encapsulation effusion, bilateral hydrosalpinx (Figure 2).

\section{Discussion}

There are various types of pelvic tumors with different morphologies which derive from different locations and growth patterns. These differences in morphological and structural characteristics can be used as the basis for tumor diagnosis (7). The diagnosis of pelvic tumors is mainly based on imaging technologies, including ultrasound, computed tomography (CT), and magnetic resonance imaging (MRI). However, MRI is rarely used clinically due to its high cost and long examination time, while CT examination is also limited by its relatively high expense. Therefore, ultrasonography is a common method for clinical examination of female pelvic gynecological malignancies (8). In vaginal ultrasound in particular, the probe can almost 

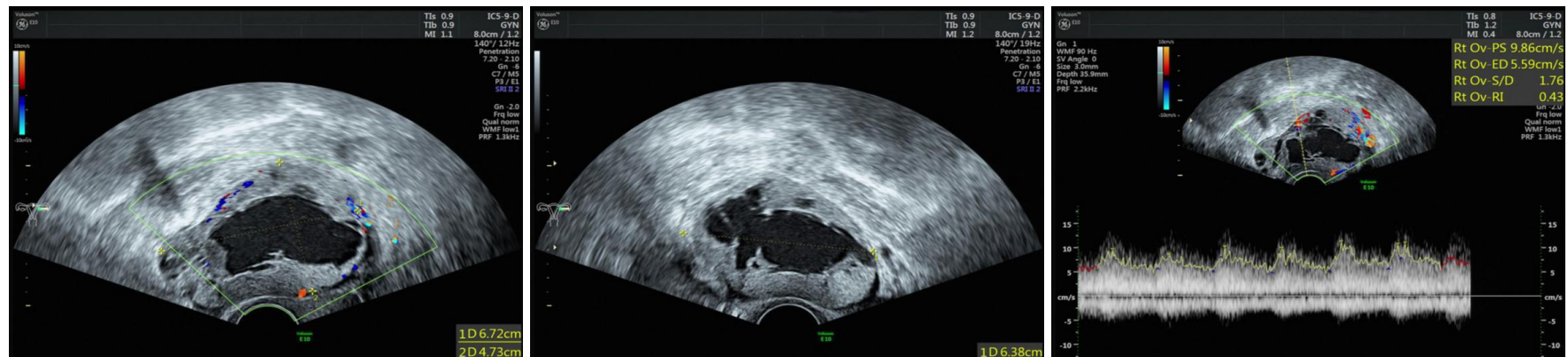

Figures 1 Images of ovarian serous adenocarcinoma, moderately differentiated. Physical examination of a 45-year-old female patient revealed a left accessory mass that had persisted for over 1 month. Ultrasound results showed that the solid echo range of the multilocular septum was about $6.7 \times 6.4 \times 4.7 \mathrm{~cm}^{3}$ in the left accessory area, with a clear boundary and irregular morphology. The maximum cyst area was $5.2 \times 3.4 \times 2.5 \mathrm{~cm}^{3}$ with poor sound transmission. Color Doppler flow imaging (CDFI) revealed visible blood flow signal in the physical area; $\mathrm{RI}=0.43$.
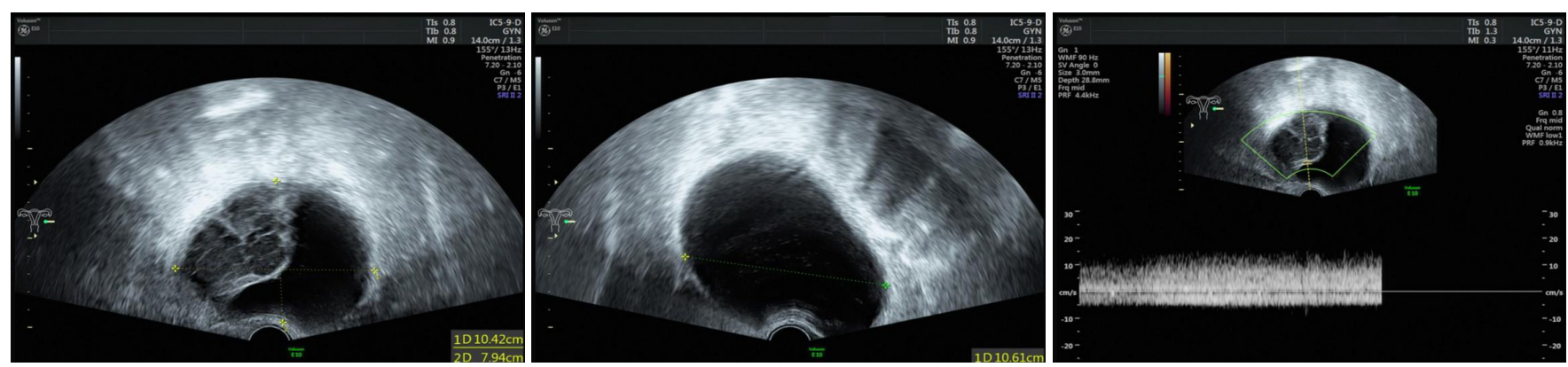

Figures 2 Images of pelvic encapsulation effusion, bilateral hydrosalpinx. The patient was a 44-year-old woman who complained of dull pain in her lower abdomen for 20 years, accompanied by menstrual changes and frequent urination for 2 months. Ultrasound images showed that there was no echo in the left accessory area, with a size of $10.4 \times 10.6 \times 7.9 \mathrm{~cm}$, and local sound transmission difference. Color Doppler flow imaging (CDFI) revealed a blood flow signal on the septum, indicating the venous spectrum.

directly touch the organ that needs to be scanned, therefore shortening the distance between the probe and the organ to be examined. Due to the relatively loose structure of the vagina, the operating physician can also improve the image resolution. In cases where the patient has excessive intestinal gas or is obese, vaginal ultrasound detection can avoid the interference of intestinal gas or the signaling attenuation of the abdominal wall fat layer, and then clearly show the tumor size, shape, and physical properties of the tumor $(9,10)$. The borders of benign pelvic tumors are often clear and have a complete capsule, while malignant tumors usually have an irregular shape and lack a complete capsule. Furthermore, benign tumors are more common in cystic lumps while malignant tumors are more common in solid or cyst-solid lumps. The results of this study are consistent with those already reported in the above-mentioned literature.
Studies by Hyde (11) and others have pointed out that malignant pelvic tumors are often accompanied by ascites. It is generally believed that ascites is secreted by tumor cells planted on the surface of the tumor or transferred to the peritoneum, and the decomposition products of the peritoneum when it is stimulated. And the amount of ascites is reported to be positively correlated with the degree of tumor malignancy (12). In this study, more than half of the malignant tumors were accompanied by ascites, while only about $2 \%$ of the patients with benign tumors were accompanied by ascites, indicating that if there is ascites of the pelvic tumor detected through ultrasound, the possibility of the tumors being malignant should be considered. However, even if no evidence of ascites is found, the possibility of malignancy cannot be ruled out, and further investigation is still required.

TVCDU detection is an imaging technology that 
combines two-dimensional ultrasound and color Doppler ultrasound, and shows certain advantages in observing tumor blood flow distribution and morphology (13). Benign tumors have small atypia, few new blood vessels, a high $\mathrm{RI}$, and most of them grow slowly. In contrast, malignant tumors grow rapidly and have many new blood vessels. The appearance of a large number of arteriovenous anastomoses and the lack of muscle tissue components in the vessel wall lead to a reduction of vascular resistance. The distribution of malignant tumors is characterized by multivessel diffuse distribution inside the septum or solid components, while the number of blood vessels in benign tumors is lower than that of malignant tumor, with the blood vessels of benign tumors being mainly located around the lesion. Benign tumors may also be characterized by a lack of blood flow $(14,15)$. Celiesiute et al. (16) and other researchers have found that benign and malignant pelvic tumors have their own characteristics in terms of blood vessel formation and blood supply. In addition, because the distribution of blood flow resistance shows a certain pattern, observing the changes in blood flow resistance can reflect the malignant characteristic of tumors.

In addition to TVCDU detection, TACDU detection has commonly been used in clinic, but TACDU detection requires the patient to hold their urine and fill their bladder, which may be difficult for the patient and is not conducive to procedures in the emergency department (17). Furthermore, the resolution of TACDU detection is low, and it is difficult to clearly show the more subtle lesions. Moreover, the accuracy of transabdominal diagnosis could be affected by intestinal gas, the intestinal tract, and scar tissue $(18,19)$. Transvaginal ultrasonography with high diagnostic accuracy, is a valuable method in compensating for the shortfalls of abdominal ultrasound. According to the results of this study, TVCDU detection is more effective in the diagnosis of benign and malignant pelvic tumors, and has the advantages of simple operation, reproducibility, and low cost, especially in the diagnosis of obese patients or those with subtle lesions, which is consistent with the findings reported by Leonardi et al. (20).

In summary, the sensitivity and accuracy of the differential diagnosis of benign and malignant pelvic tumors in women at childbearing age by TVCDU detection is significantly higher than those of TACDU. The combined application of CDUS and RI can further improve the diagnostic accuracy of pelvic tumors.

\section{Acknowledgments}

Funding: None.

\section{Footnote}

Reporting Checklist: The authors have completed the STARD reporting checklist. Available at http://dx.doi.org/10.21037/ atm-20-7406

Data Sharing Statement: Available at http://dx.doi. org/10.21037/atm-20-7406

Conflicts of Interest: All authors have completed the ICMJE uniform disclosure form (available at http://dx.doi. org/10.21037/atm-20-7406). The authors have no conflicts of interest to declare.

Ethical Statement: The authors are accountable for all aspects of the work in ensuring that questions related to the accuracy or integrity of any part of the work are appropriately investigated and resolved. This study was approved by the Beijing Hospital (No. 2019BJYYEC-167-01). All procedures performed in this study involving human participants were in accordance with the Declaration of Helsinki (as revised in 2013). Individual consent for this retrospective analysis was waived.

Open Access Statement: This is an Open Access article distributed in accordance with the Creative Commons Attribution-NonCommercial-NoDerivs 4.0 International License (CC BY-NC-ND 4.0), which permits the noncommercial replication and distribution of the article with the strict proviso that no changes or edits are made and the original work is properly cited (including links to both the formal publication through the relevant DOI and the license). See: https://creativecommons.org/licenses/by-nc-nd/4.0/.

\section{References}

1. Câmara-de-Souza AB, Toyoshima MTK, Giannella ML, et al. Insulinoma: A retrospective study analyzing the differences between benign and malignant tumors. Pancreatology 2018;18:298-303.

2. Rei A. Waldman MD a, Justin Finch $M D$ a, Skin Diseases of the Breast and Nipple Part I: Benign and Malignant Tumors. J Am Acad Dermatol 2019;80:1467-81. 
3. Alsaggaf R, Wang Y, Marini-Bettolo C, et al. Benign and malignant tumors in the UK myotonic dystrophy patient registry. Muscle Nerve 2018;57:316-20.

4. Harris CJ, Lautz TB, Rowell EE. Feasibility of laparoscopic ovarian tissue cryopreservation after open abdominopelvic tumor surgery. Am J Surg 2020;220:1249-52.

5. Martre P, Codjia T, Tuech JJ, et al. Pelvic tumor fed by the superior mesenteric artery. What is your diagnosis? GIST complicating Meckel's diverticulum. J Visc Surg 2018;155:83-5.

6. Nougaret S, Nikolovski I, Paroder V, et al. MRI of Tumors and Tumor Mimics in the Female Pelvis: Anatomic Pelvic Space-based Approach. Radiographics 2019;39:1205-29.

7. Wein AJ. Re: Pelvic Organ Prolapse Surgery in Academic Female Pelvic Medicine and Reconstructive Surgery Urology Practice in the Setting of the Food and Drug Administration Public Health Notifications. J Urol 2017;197:481.

8. Huang B, Tang SK, Saw T. Enterobius vermicularis infection of the female pelvic peritoneum diagnosed by laparoscopic biopsy: 2 case reports. Pathology 2019;51:S84.

9. Gilligan LA, Trout AT, Schuster JG, et al. Normative values for ultrasound measurements of the female pelvic organs throughout childhood and adolescence. Pediatr Radiol 2019;49:1042-50.

10. Weissbart SJ, Wein AJ, Smith AL. Female Pelvic Medicine and Reconstructive Surgery-What Does Certification Mean? Curr Urol Rep 2018;19:30.

11. Hyde BJ, Byrnes JN, Occhino JA, et al. MRI review of female pelvic fistulizing disease. J Magn Reson Imaging 2018;48:1172-84.

12. Annam A. Female Pelvic Vascular Malformations. Semin Intervent Radiol 2018;35:62-8.

Cite this article as: Zhi M, Wang M, Li W, Ma L, Liu Q, Li Y, Lv Q. Reliability of the application of transvaginal color Doppler ultrasound in the identification of benign and malignant pelvic tumors in women of childbearing age. Ann Transl Med 2020;8(24):1662. doi: 10.21037/atm-20-7406
13. Iglesia, Cheryl. McGraw-Hill Education Specialty Board Review: Female Pelvic Medicine and Reconstructive Surgery. Eur J of Anaesthesiol 2017;31:918.

14. Cormio L, Mancini V, Liuzzi G, et al. Surgical management of female pelvic organ prolapse with and without urinary incontinence: A single center experience. Medicine 2017;96:e7914.

15. Ros C, Barnes D, Fervienza A, et al. P14.13: Ultrasound guided transvaginal thrombin injection to treat uterine arteries pseudoaneurysms. Ultrasound Obstet Gynecol 2017;50:200-1.

16. Celiesiute J, Gaurilcikas A, Gedgaudaite M, et al. Transvaginal ultrasound - noninvasive method for the prediction of response to concurrent chemoradiotherapy in cases of cervical cancer. Journal of Vibroengineering 2017;19:2180-7.

17. Vangoitsenhoven M, Van d BT. EP26.08: Endometroid carcinoma not to be mistaken for deep infiltrating endometriosis on transvaginal ultrasound. Ultrasound Obstet Gynecol 2017;50:383-3.

18. Rodgers JR, Hrinivich WT, Surry K, et al. A semiautomatic segmentation method for interstitial needles in intraoperative 3D transvaginal ultrasound images for high-dose-rate gynecologic brachytherapy of vaginal tumors. Brachytherapy 2020;19:659-68.

19. Petrone M, Bergamini A, Tateo S, et al. Transvaginal ultrasound in evaluation and follow-up of ovarian granulosa cell tumors. Int J Gynecol Cancer 2020;30:1384-9.

20. Leonardi M, Martins WP, Espada M, et al. Prevalence of negative sliding sign representing pouch of Douglas obliteration during pelvic transvaginal ultrasound for any indication. Ultrasound Obstet Gynecol 2020;56:928-33.

(English Language Editor: J. Gray) 